collecting system. Early in one's experience, a catheterized approach may be beneficial for maximizing successful access with minimal complications. Once a level of comfort with percutaneous anatomy is achieved, a noncatheterized approach may be a natural evolution to performing percutaneous nephrolithotomy.

A larger randomized study would assist in determining the value of pre-procedure catheter placement for obtaining optimal access to the collecting system. As there are a limited number of published randomized studies on access relating to percutaneous nephrolithotomy, the authors should be congratulated on their contribution."

Dr. Ravi Munver

Chief, Minimally Invasive Urologic Surgery Hackensack University Medical Center Hackensack, New Jersey, USA E-mail:rmunver@humed.com

\title{
Re: Antibiotic Resistance and Trend of Urinary Pathogens in General Outpatients from a Major Urban City
}

\author{
Carlos R. Kiffer, Caio Mendes, Carmen P. Oplustil, Jorge L. Sampaio
}

Section of Microbiology, Fleury Institute, Sao Paulo, SP, Brazil

Int Braz J Urol, 33: 42-49, 2007

To the Editor:

Investigations of antibiotics susceptibility profiles on urinary tract infections in outpatients in São Paulo, Brazil, were aimed to recommend the ideal empirical therapeutic recipe in suspected cases of urinary tract infections (1). That had been a commendable exercise through for the maximal utility of any retrospective data in clinical practice, it would have been better to evaluate identical profiles in hospitalized patients as well. Clinicians would be treating patients during and after hospitalization. A watch is being continued on combined susceptibility pattern in a private, tertiary care hospital in the Indian capital metropolis.

Effective October 2004, susceptibility data was compiled at Sant Parmanand Hospital, a 140- bedded tertiary care hospital catering to population in the capital and adjoining townships. The antimicrobial susceptibility on urinary isolates would be determined using disk diffusion method for amoxicillin-clavulanic acid, ampicillin-sulbactam, cefaclor, cefuroxime, ceftriaxone, ceftazidime, cefotaxime, ceftizoxime, amikacin, gentamicin, netilmicin, ciprofloxacin, ofloxacin, pefloxacin, norfloxacin, nalidixic acid, chloramphenicol and nitrofurantoin. The outstanding antimicrobials were selected based on the previous quarterly susceptibility pattern, from October to December 2004. Based on the picture in 130 isolates namely, E. coli, 103, Klebsiella species, 20, Proteus species, 13 and Pseudomonas species 11, data was computed to 
determine a cut-off value of $75 \%$ or more in-vitro susceptible. Antimicrobials selected were categorized into oral or parenteral antimicrobials (2). The oral recipe identified was of amoxicillin-clavulanic acid, chloramphenicol and nitrofurantoin, the parenteral recipe of amikacin, netilmicin and ceftazidime. Subsequently, similar data from inpatients and outpatients for the every quarter was assessed in

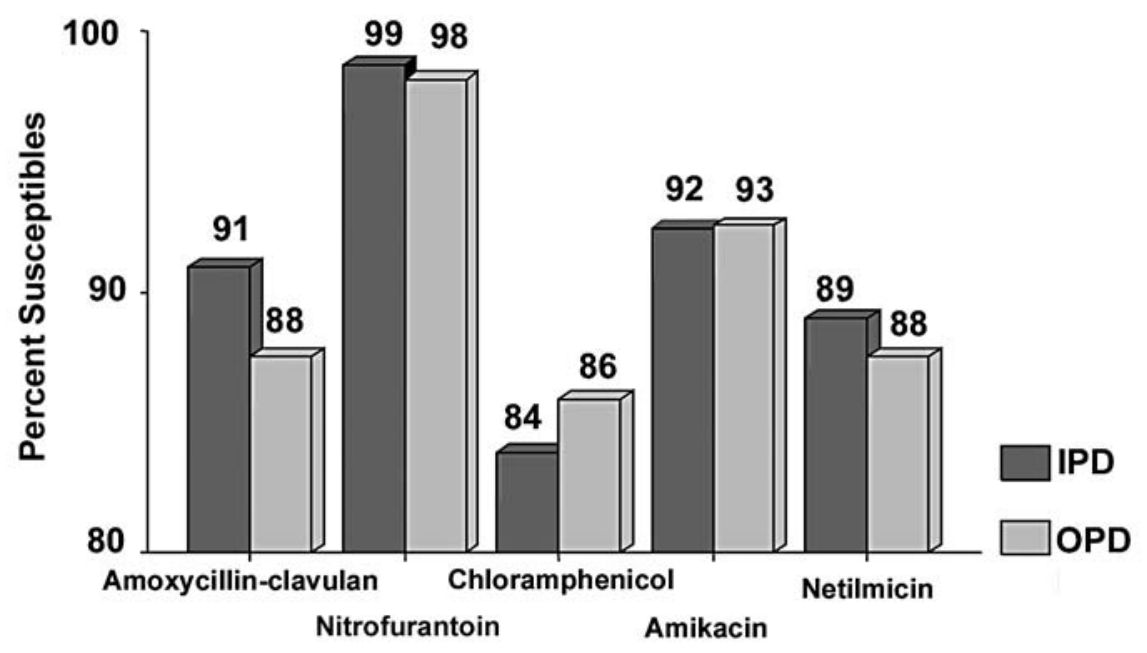

Antibiotics

Figure 1 - Outpatients (OPD) and inpatients (IPD) susceptibility profile.

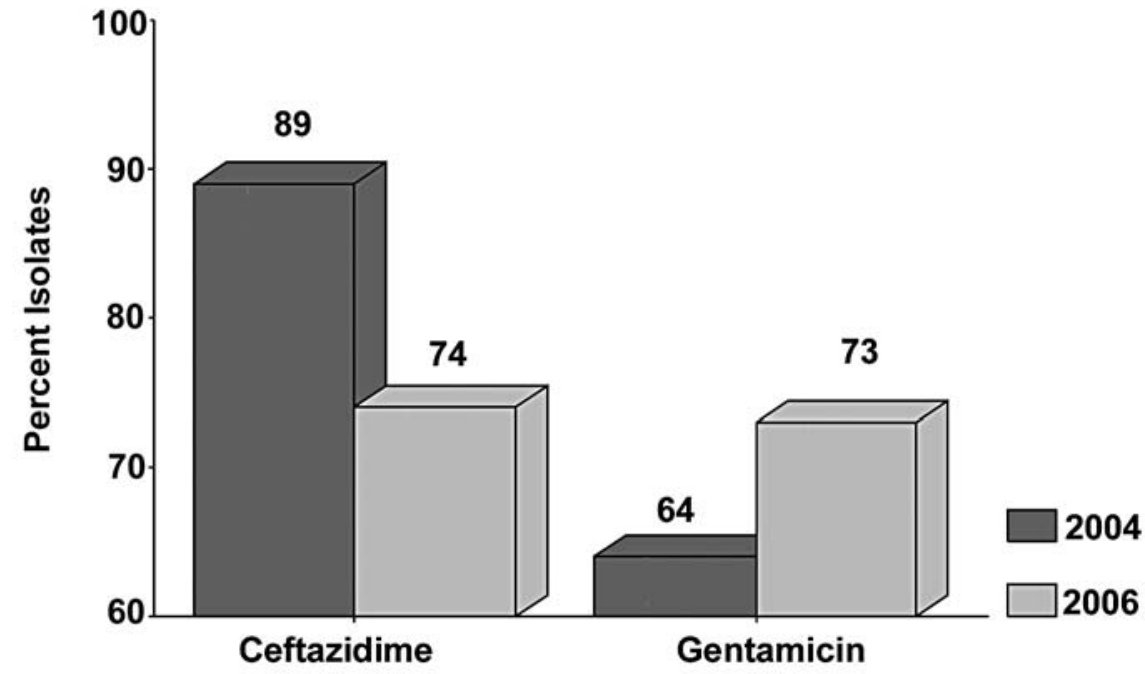

Antibiotics

Fisher's p value $0.0007,0.0195$, respectively

Figure 2 - Altered susceptibility profile. 
relation to the retrospective data. There was no significant change during 2005.

The susceptibility pattern during the 12 month duration from December 2005 to 2006 from outpatients and inpatients was not all diverse. Isolates had included E. coli, 1209 strains, Klebsiella, 650, Proteus, 379, Pseudomonas, 32 and Enterococcus feacalis, 6 strains. The contribution of inpatients was of E. coli, 269 strains, Klebsiella, 147, Proteus, 44, Pseudomonas, 12 and Enterococcus feacalis, 2 only. There was no significant difference for amoxicillinclavulanic acid, chloramphenicol, nitrofurantoin, amikacin and netilmicin susceptibility among inpatient and outpatient isolates (Figure-1). There was a significant reduction in the prevalence of isolates susceptible to ceftazidime, accompanied by an increase in isolates susceptible to gentamicin (Figure-2).

The clinical course in neonates with urinary tract infections treated with short-term intravenous antibiotic followed by oral treatment is highly favorable, both in short and long terms (3). Selection of appropriate intravenous and oral antibiotics against isolates in local circulation should be a reality through updates to the susceptibility profile of urinary isolates (1) encountered among patients seeking treatment in the hospital premises (2). That would also encourage frequent dialogues amongst clinical microbiology personnel and those responsible for patient care and guarantee better empirical recipe in urinary tract infections.

\section{References}

1. Kiffer CR, Mendes C, Oplustill CP, Sampaio JL: Antibiotic resistance and trend of urinary pathogens in general outpatients from a major urban city. Int Braz J Urol. 2007; 33: 42-9.

2. Arya SC, Agarwal N, Agarwal S: Straightforward representation of antimicrobial chemotherapeutics susceptibility profiles in a private tertiary care hospital. J Infect. 2005; 51: 333-5.

3. Magin EC, Garcia-Garcia JJ, Sert SZ, Giralt AG, Cubells CL: Efficacy of Short-Term Intravenous Antibiotic in Neonates With Urinary Tract Infection. Pediat Emerg Care. 2007; 23: 83-6.

\author{
Dr. Subhash C. Arya \\ Dr. Nirmala Agarwal \\ Sant Parmanand Hospital \\ Delhi, India
}

Email:subhashji@hotmail.com

\title{
Re: Wet Heat Exposure: A Potentially Reversible Cause of Low Semen Quality in Infertile Men
}

\author{
Shai Shefi, Phiroz E. Tarapore, Thomas J. Walsh, Mary Croughan, Paul J. Turek \\ Departments of Urology, Obstetrics, Gynecology and Reproductive Science, and Epidemiology and \\ Biostatistics, University of California San Francisco, San Francisco, California, USA
}

Int Braz J Urol, 33: 50-57, 2007

To the Editor:

Dry and wet heat differ mainly in the speed of energy transfer, as heat transfer by convection and contact in water is increased than in air, and because heat dissipation by sweat evaporation is blocked into 